\title{
CPTAC Liver Hepatocellular Carcinoma Baseline Form
}

National Cancer Institute

\section{Source}

National Cancer Institute. CPTAC Liver Hepatocellular Carcinoma Baseline Form. NCI

Thesaurus. Code C157529.

Terminology used in support of the data collection efforts of the Clinical Proteomic

Tumor Analysis Consortium (CPTAC) with the focus on Liver Hepatocellular Carcinoma Baseline Form data. 